\title{
FUNCIONALIZAÇÃO ÁCIDA DE CARVÃO ATIVADO DE DENDÊ NA REMOÇÃO DE PARACETAMOL
}

\author{
B. A. MASSIAS ${ }^{2}$, H.H.C. LIMA ${ }^{1}$, R. C. FERREIRA ${ }^{1}$, P. A. ARROYO ${ }^{1}$, M. A. S. D. BARROS ${ }^{1}$ \\ ${ }^{1}$ Universidade Estadual de Maringá, Departamento de Pós-Graduação em Engenharia Química \\ ${ }^{2}$ Universidade Estadual de Maringá, Departamento de Engenharia Química \\ *e-mail: hugolima22@ @otmail.com
}

\begin{abstract}
RESUMO
No intuito de investigar as características de adsorção de paracetamol em carvão ativado, o carvão ativado de dendê foi tratado com $1 \mathrm{~mol} \mathrm{~L}^{-1}$ de ácido sulfúrico a $40^{\circ} \mathrm{C}$. As propriedades texturais como área de superfície específica, volume de poros, volume de microporos e diâmetro médio de poro foram realizadas para caracterizações texturais e para caracterização química a titulação de Boehm, ponto de carga foram empregados e espectroscopia no infravermelho. Modelos cinéticos de pseudo-primeira ordem e pseudo-segunda ordem foram empregados para expressar o mecanismo de adsorção. Para correlacionar os dados de equilíbrio foram ajustadas as isotermas de Langmuir e de Freundlich. Após a funcionalização ácida, ocorreu o decréscimo da área de superfície específica e do volume de poros. A superfície do carvão após a funcionalização apresentou maior quantidade de grupos ácidos. $\mathrm{O}$ modelo cinético de pseudo-segunda ordem e a isoterma de Langmuir representam melhor os dados experimentais cinéticos e de equilíbrio, tanto para CAD como para CADSO4 assim caracterizando adsorção em monocamada.
\end{abstract}

Palavras chaves: Carvão Ativado, Paracetamol, Tratamento Ácido, Adsorção;

\section{INTRODUÇÃO}

Os fármacos fazem parte uma classe emergente de contaminantes, os quais, estão recebendo atenção considerável devido aos impactos ambientais que causam (FENT, WESTON e CAMINADA, 2006).

A ocorrência destes contaminantes no ambiente pode acontecer através de diferentes fontes, tais como, a excreção de parte dos fármacos não absorvidos pelo corpo humano, o descarte inadequado de medicamentos não utilizados, e até mesmo durante a produção dos fármacos (VILLAESCUSA et al., 2011).

Como são frequentemente encontrados em águas residuais e em estações de tratamento de esgoto em níveis de $n g \mathrm{~L}^{-1}$ e $\mu \mathrm{g}$
$\mathrm{L}^{-1}$, os processos convencionais das estações de tratamentos não são capazes de remover ou degradar esses compostos, já que apresentam estrutura química estável e não são biodegradáveis (DUCLAUX, et al. 2013; MOREIRA, et al. 2010; FENT, WESTON e CAMINADA, 2006).

Os fármacos são detectados com maior frequência, pois os medicamentos como o paracetamol, além de serem empregados para o tratamento de dores leves e moderadas, podem ser comercializados sem prescrição médica (BARCELÓ et al. 2008). Assim como outros medicamentos, o paracetamol é absorvido pelo organismo e cerca de 58 a $68 \%$ do fármaco é eliminado através da urina (MUIR et al.1997). 
Embora os resíduos fármacos não estejam regulamentados nas diretrizes de água em todo mundo, há uma crescente preocupação da comunidade cientifica em relação à saúde pública e ao impacto ambiental provocados por essas substâncias como reportados nos estudos de DUCLAUX, et al. 2013; MASTRAGOSTINO et al. 2009; MESTRE et al.2014, BACCAR et al.2012.

Segundo VILLAESCUSA et al. (2011), para a remoção de fármacos das águas residuais deve-se utilizar tecnologias avançadas, tais como a ozonização, processo de oxidação avançada, osmose inversa, filtração por membrana e adsorção em carvão ativado.

A adsorção tem-se mostrado eficiente para a remoção de diversos fármacos, e dentre eles, do paracetamol. De fato, isto acontece devido à forte interação entre a composição da superfície e as propriedades químicas do carvão mesmo em diferentes temperaturas e valores de $\mathrm{pH}$ (TERZYK e RYCHLICKI, 2000).

Como há diferentes origens de carvão ativado tem-se investigado aqueles que possam propiciar elevados índices de remoção e que sejam de fácil obtenção e de baixo custo. Além disso, diversos estudos indicam que a funcionalização do carvão altera suas propriedades tanto texturais quanto químicas e podem modificar o mecanismo de adsorção (COUTO-JR, et al. 2015).

Neste contexto, o objetivo do presente trabalho foi verificar a remoção de paracetamol em carvão de origem nacional, obtido a partir do coco de dendê. Também foi avaliada a alteração da adsorção do fármaco com o carvão funcionalizado com ácido sulfúrico.

\subsection{MATERIAS E MÉTODOS}

\subsection{Preparação do Carvão Ativado}

O carvão ativado de coco de dendê (CAD) cedido pela empresa Bahiacarbon Agro Ind. (Bahia, Brasil) foi triturado e peneirado com diâmetro médio dos poros de

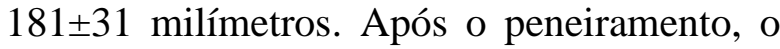
CAD foi lavado com água deionizada a $80^{\circ} \mathrm{C}$ e seco em estufa de circulação de ar a $45^{\circ} \mathrm{C}$. Amostras de $\mathrm{CAD}$ foram funcionalizadas com $1 \mathrm{~mol} \mathrm{~L}^{-1}$ de $\mathrm{H}_{2} \mathrm{SO}_{4}$ durante 6 horas a $40^{\circ} \mathrm{C}$, lavado novamente com água deionizada dando origem, portanto à amostra CADSO4 (LI, et al. 2013 adaptado).

\subsection{Caracterização dos Carvões Ativados}

\subsubsection{Adsorção e Dessorção de $\mathrm{N}_{2}$}

As análises de adsorção e dessorção forma feitas utilizando o equipamento Micromeritics, modelo ASAP 2020. As amostras foram submetidas a vácuo de 2 $\mu \mathrm{mHg}$ e temperatura de $300{ }^{\circ} \mathrm{C}$ durante 4 horas afim de se garantir ausência de contaminantes. A adsorção e dessorção de nitrogênio foi realizada a temperatura de 77 K. A partir dos dados de adsorção e dessorção foi possível calcular as propriedades texturais do carvão ativado, tais como, área específica, distribuição de volume de poros, diâmetro médio dos poros e o volume de microporos.

\subsubsection{Titulação de Boehm}

Para quantificação dos grupos funcionais presente na superfície dos carvões ativados, tais como lactona (R-OCO), fenol (R-OH), carboxílicos (R-COOH), foi quantificado utilizando método de BOEHM (1994)

\subsubsection{Ponto de Carga Zero}

A determinação do ponto de carga zero $\left(\mathrm{pH}_{\mathrm{pcz}}\right)$ do carvão ativado foi baseada na metodologia proposta REGABULTO e JAIME (2004) em que PCZ representa o valor do $\mathrm{pH}$ quando a carga da superfície do carvão ativado é zero. $\mathrm{O} \quad \mathrm{pH}_{\mathrm{pcz}}$ depende das propriedades químicas e eletrônicas dos 
grupos superficiais do carvão ativado (SHAKAR et al., 2014).

2.2.4 Espectroscopia no Infravermelho com transformada Fourier

Para obtenção dos espectros no infravermelho utilizou-se o espectrofotômetro da BRUKER, modelo Vertex 70v. Os carvões ativados foram secos em estufa por 24 horas a $100^{\circ} \mathrm{C}$. Posteriormente, os carvões ativados foram macerados com $\mathrm{KBr}$ numa proporção de $99,9 \%$ de $\mathrm{KBr}$ e $0,1 \%$ de carvão. O espectro de leitura variou na faixa de 4000 a $400 \mathrm{~cm}^{-1}$ com resolução de $2 \mathrm{~cm}^{-1}$ e 128 varreduras.

\subsection{Ensaios Cinéticos de Adsorção}

Para os ensaios de cinética de adsorção, $20 \mathrm{~mL}$ de solução de paracetamol a $50 \mathrm{mg} \mathrm{L}^{-1}$ foram misturados com $10 \mathrm{mg}$ de cada amostra de carvão ativado. Os sistemas assim formados foram submetidos à agitação em banho Dubnoff a $150 \mathrm{rpm}$ a $25^{\circ} \mathrm{C}$. Após tempos pré-definidos, os sistemas foram filtrados $e$ as concentrações iniciais e residuais de paracetamol foram analisadas no espectrofotômetro de UV-VIS (UVmini-1240 Shimadzu) no comprimento de onda de $245 \mathrm{~nm}$ (HOTZA et al.2014).

Os ensaios cinéticos foram realizados em triplicata e a quantidade adsorvida de paracetamol foi calculada pelo balanço material.

$q_{\mathrm{t}}=\frac{\left(c_{0}-c_{\mathrm{t}}\right)_{v}}{m}$

A cinética de adsorção do paracetamol foi modelada usando expressões matemáticas que incluem a cinética de pseudo-primeira ordem (LAGERGREN, 1898), cinética de pseudo-segunda ordem (McKAY e HO, 1999).

A equação de cinética de pseudoprimeira ordem descrita por Lagergren é expressa pela Equação 2. $q_{t}=q_{\theta q}\left(1-e^{-k_{1} t}\right)$

A equação de cinética de pseudosegunda ordem é expressa pela Equação 3.

$q_{t}=\frac{q_{s q}{ }^{2} K_{2} t}{1+q_{g q} K_{2} t}$

\subsection{Ensaios de Isotermas de Adsorção}

Para os ensaios de equilíbrio, 7 a $40 \mathrm{mg}$ de CAD ou CADSO4 foram adicionados a $20 \mathrm{~mL}$ de solução de paracetamol de concentração $50 \mathrm{mg} . \mathrm{L}^{-1}$. Os ensaios foram realizados em banho Dubnoff e também a $25^{\circ} \mathrm{C}$, sob agitação de $150 \mathrm{rpm}$ em triplicata. Após atingir o tempo de equilíbrio determinado pela cinética, as amostras foram filtradas, a concentração final e a quantidade de paracetamol adsorvido obtida pela Equação 1 .

Os dados experimentais foram analisados aplicando os modelos de isoterma de Langmuir (LANGMUIR, 1918), Freundlich (FREUNDLICH, 1906).

$\mathrm{O}$ modelo de Langmuir, descrito pela equação 4 , parte do pressuposto de que a atração entre o adsorbato e a superfície do adsorvente baseia-se em forças de van der Waals, ocorrendo adsorção em sítios específicos do adsorvente sem a interações entre as moléculas adsorvidas, formando uma monocamada com energia de adsorção envolvida igual para todos os sítios de adsorção (Langmuir,1918).

$q_{e}=\frac{q_{\max } K_{L} c_{e q}}{1+K_{L} c_{e q}}$

O modelo empírico da isoterma de Freundlich, expressa pela equação 5, considera o sólido heterogêneo e a existência da formação de multicamada os quais possuem diferentes energias adsortivas, assim não ocorrendo a saturação da superfície (FREUNDLICH, 1906). 
$q_{e}=K_{F} c_{e q}^{\frac{1}{n}}$

\section{RESULTADOS E DISCUSSÕES}

\subsection{Caracterização dos Carvões Ativados}

As isotermas de adsorção e dessorção de nitrogênio, Figura 1, para CAD e CADSO4 são bastante semelhantes. Ambas as isotermas são do tipo I de acordo com a classificação da IUPAC, ou seja, os adsorventes são microporosos com contribuição de mesoporos, já que possuem histerese do tipo $\mathrm{H} 4$, em pressões relativas acima de 0,5 .

Figura 1- Isotermas de adsorção e dessorção de $\mathrm{N}_{2}$ para as amostras de CAD e CADSO4.

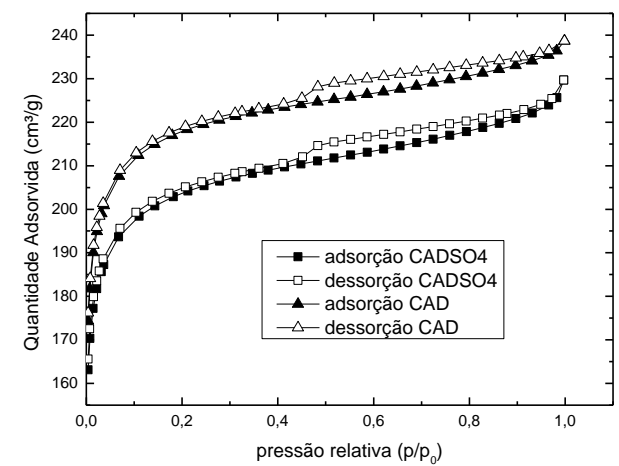

Fonte: Autor (2015)

A Figura 2 apresenta a distribuição de tamanho de poros. Observa-se que o tratamento ácido promoveu diminuição do volume de microporos. De fato, pelo método $\mathrm{BJH}$, o diâmetro médio caiu de $34,50 \AA$ para CAD e para $13,60 \AA$ para CADSO4, o que está coerente com as isotermas apresentadas na Figura 1, em que CADSO4, adsorveu menor quantidade de $\mathrm{N}_{2}$ e possui menor área.

A Tabela 1 resume algumas das características texturais dos adsorventes. A funcionalização diminuiu a área superficial do carvão. Após a funcionalização, ocorreu um decréscimo do volume total de poros de
CADSO4 $\left(0,355 \mathrm{~cm}^{3} \mathrm{~g}^{-1}\right)$. No CAD $(0,369$ $\mathrm{cm}^{3} \mathrm{~g}^{-1}$ ) apresenta uma contribuição de $77 \%$ de microporos, e no CADSO4 a contribuição é de $74 \%$, portanto a funcionalização não afetou a contribuição de microporos e mesoporos.

Figura 2- Distribuição de Tamanho de Poros para amostras de CAD e CADSO4

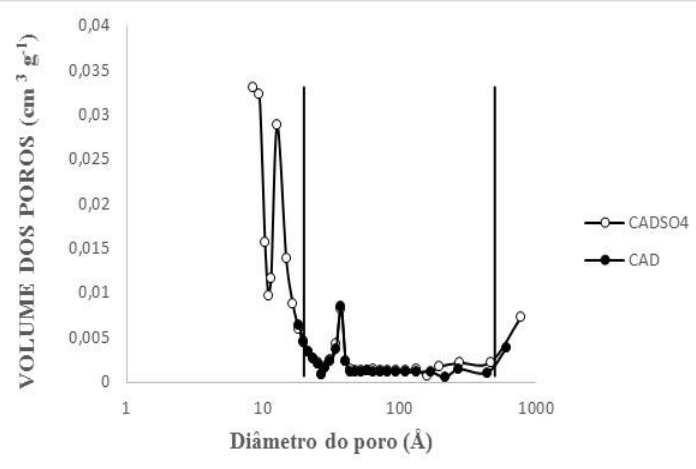

Fonte: Autor (2015)

Tabela 1 - Caracteristicas Texturais de CAD e CADSO4

\begin{tabular}{ccc}
\hline Amostra & CAD & CADS04 \\
\hline$A_{\text {BET }}\left(\mathrm{m}^{2} \mathrm{~g}^{-1}\right)$ & $671 \pm 22$ & $629 \pm 20$ \\
$\mathrm{~V}_{\text {total }}\left(\mathrm{cm}^{3} \mathrm{~g}^{-1}\right)$ & 0,369 & 0,355 \\
$\mathrm{~V}_{\text {meso }}\left(\mathrm{cm}^{3} \mathrm{~g}^{-1}\right)$ & 0,082 & 0,089 \\
$\mathrm{~V} \alpha$ micro $\left(\mathrm{cm}^{3} \mathrm{~g}^{-1}\right)^{*}$ & 0,287 & 0,266 \\
$\mathrm{~V} \alpha_{\text {ultra }}\left(\mathrm{cm}^{3} \mathrm{~g}^{-1}\right)^{*}$ & 0,207 & 0,207 \\
$\mathrm{~V} \alpha_{\text {super }}\left(\mathrm{cm}^{3} \mathrm{~g}^{-1}\right)^{*}$ & 0,08 & 0,071 \\
D. Poro $(\mathrm{BJH})(\AA)$ & 34,50 & 13,60 \\
\hline
\end{tabular}

Fonte: Autor (2015) *método de $\alpha_{\mathrm{s}}$

Segundo Li et al. (2013) o decréscimo da área pode estar relacionado a erosão na parte interna do carvão ativado e da oxidação da superfície, causado pela presença de $\mathrm{H}_{2} \mathrm{SO}_{4}$. A diminuição do volume total de poros está relacionada a fatores, tais como, o bloqueio da entrada dos poros por grupos funcionais contendo oxigênios ou pela destruição das paredes pelo tratamento oxidante e também pelo alargamento dos poros (LIU, LIU, e LI, et al. 2011).

$\mathrm{O}$ valor de $\mathrm{pH}_{\mathrm{pcz}}$ do $\mathrm{CADSO} 4\left(\mathrm{pH}_{\mathrm{pcz}}=\right.$ $3,61)$ é inferior comparado com o CAD ( $\mathrm{pH}_{\mathrm{pcz}}$ $=6,6$ ). Tal fato é consequência da maior quantidade de grupos funcionais ácidos e 
menor quantidade de grupos básicos presentes na superfície do CADSO4, como pode ser visto pelos resultados da titulação do método de Boehm apresentados na Tabela 2.

Tabela 2- Titulaçãode Boehm de CAD e CADCL

\begin{tabular}{ccc}
\hline Amostra & CAD & CADCL \\
\hline GC (meq g $\left.{ }^{-1}\right)$ & 1,096 & 2,637 \\
GF (meq g $\left.{ }^{-1}\right)$ & 0 & 1,713 \\
GL (meq g $\left.{ }^{-1}\right)$ & 0,053 & 0,424 \\
GTA (meq g $\left.{ }^{-1}\right)$ & 1,149 & 4,773 \\
GB (meq g $\left.{ }^{-1}\right)$ & 0,768 & 0,449 \\
\hline
\end{tabular}

Em geral, a titulação de Boehm está de acordo com as medidas do $\mathrm{pH}_{\text {pcz }}$. O CAD contém grupos superficiais tais como, grupos carboxílicos (GC, 1,096 meq g ${ }^{-1}$ ) e lactônicos (GL, 0,053 meq $\mathrm{g}^{-1}$ ) e grupos básicos (GB, 0,768 meq $^{-1}$ ) e após a funcionalização há a eliminação de impurezas inorgânicas e o aumento dos grupos ácidos da superfície do carvão (NEVSKAIA et al., 2004). Assim sendo, a titulação de Boehm torna-se mais sensível à detecção destes grupos, como evidenciado no CADSO4, em que ocorre o aumento dos grupos carboxílicos (GC, 2,637 meq $\mathrm{g}^{-1}$ ) e lactônicos (GL, 0,424 meq $\mathrm{g}^{-1}$ ) além da presença de grupos fenólicos (GF, 1,713 meq $\mathrm{g}^{-1}$ ) e diminuição dos grupos básicos (GB, 0,449 meq g $\left.{ }^{-1}\right)$.

$\mathrm{O}$ fato dos grupos carboxílicos aumentarem mais do que dos outros grupos ácidos está relacionada à capacidade de oxidação do ácido utilizado na funcionalização. Portanto, quanto maior a oxidação, maior será a quantidade de grupos carboxílicos presentes na superfície do carvão (HUANG, LI e CHEN, 2008; LIU, LIU, e LI, et al. 2011).

$\mathrm{O}$ espectro no infravermelho para os dois adsorventes estão apresentados na Figura 3. Os espectros no infravermelho para os adsorventes são semelhantes, na região de $3437 \mathrm{~cm}^{-1}$ referente ao estiramento da ligação $\mathrm{O}-\mathrm{H}$, em $1570 \mathrm{~cm}^{-1}$ referente ao estiramento da dupla ligação $\mathrm{C}=\mathrm{C}$, em $1136 \mathrm{~cm}^{-1}$ referente ao estiramento de C-O-C. Após a funcionalização com ácido sulfúrico, observase um pico na região entre $1142-1116 \mathrm{~cm}^{-1}$ que indica a introdução de grupos sulfônicos (HUANG, LI e CHEN, 2008; LIU, LIU, e LI, et al. 2011; Li, et al. 2013).

Figura 3- Espectro no infravermelho para CAD e CADSO4

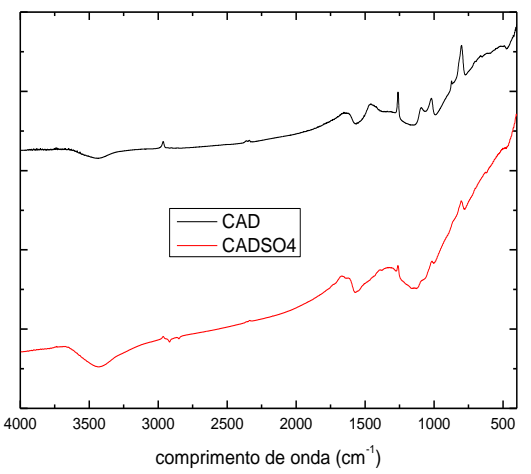

Fonte: Autor (2015)

\subsection{Cinética de Adsorção de Paracetamol}

A cinética de adsorção de paracetamol para os dois carvões ativado, CAD e CADSO4 foram realizados para estudar o mecanismo de adsorção do paracetamol. Os resultados estão expostos nas figuras 4 e 5 .

Nas figuras 4 e 5 pode ser visto que o sistema atingiu o equilíbrio em 240 minutos e que no início ocorreu uma adsorção rápida, devido à maior disponibilidade de sítios na superfície dos carvões ativados.

Figura 4- Cinética de adsorção de CAD

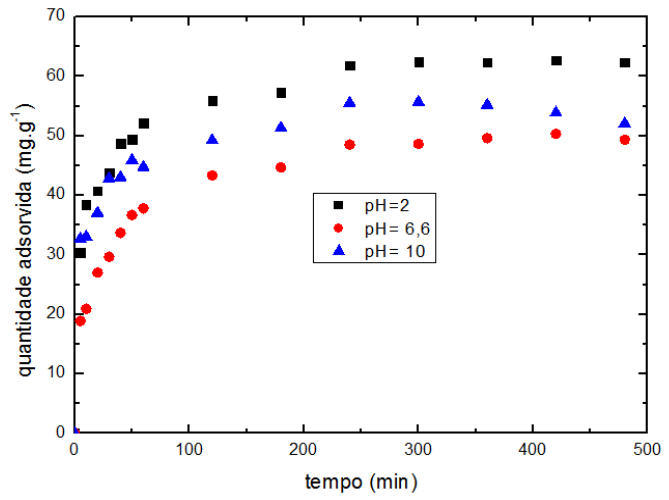


Fonte: autor (2015)

Figura 5- Cinética de adsorção de

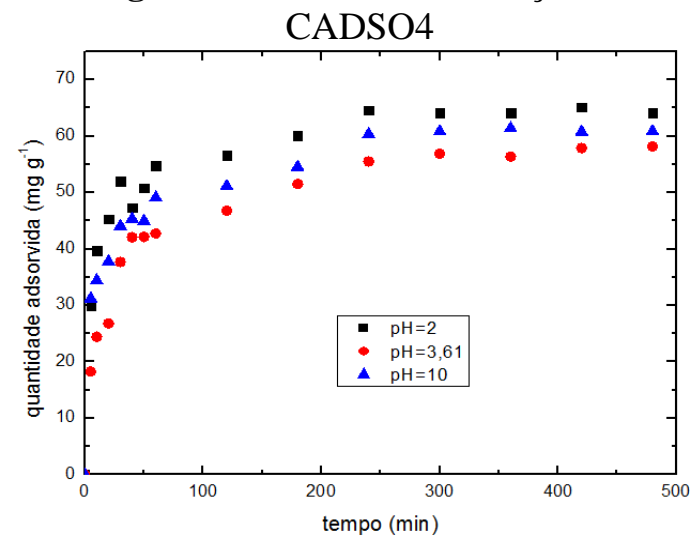

Fonte: autor (2015)
Os dados experimentais foram ajustados aos modelos cinéticos de pseudoprimeira ordem e pseudo-segunda ordem e os ajustes estão dispostos na Tabela 4 .

$\mathrm{Na}$ tabela 4, nota-se que para ambos os carvões o modelo cinético de pseudo-segunda ordem representou melhor os dados experimentais, pois apresentaram maior valor de $\mathrm{R}^{2}$. Além disso, os parâmetros obtidos possuíram valores baixos de desvio e a quantidade adsorvida no equilíbrio assemelham se aos resultados experimentais apresentados na Tabela 3 .

O modelo cinético de pseudo-segunda ordem foi aplicado com sucesso para a

Tabela 4- Parâmetros cinéticos para CAD e CADSO4

\begin{tabular}{|c|c|c|c|c|c|}
\hline $\begin{array}{l}\text { Carvão } \\
\text { Ativado }\end{array}$ & Modelo Cinético & Parâmetros & $\mathrm{pH}=2$ & $\mathrm{pH}=\mathrm{pH}_{\mathrm{pcz}}$ & $\mathrm{pH}=10$ \\
\hline \multirow{6}{*}{ CAD } & \multirow{3}{*}{$\begin{array}{c}\text { Pseudo-primeira } \\
\text { ordem }\end{array}$} & $\mathrm{q}_{\mathrm{eq}}\left(\mathrm{mg} \mathrm{g}^{-1}\right)$ & $57,64 \pm 2,11$ & $46,87 \pm 1,64$ & $50,30 \pm 1,81$ \\
\hline & & $\mathrm{K}_{1}\left(\min ^{-1}\right)$ & $0,082 \pm 0,017$ & $0,0328 \pm 0,005$ & $0,115 \pm 0,026$ \\
\hline & & $\mathrm{R}^{2}$ & 0,8558 & 0,9086 & 0,8379 \\
\hline & \multirow{3}{*}{$\begin{array}{l}\text { Pseudo-segunda } \\
\text { ordem }\end{array}$} & $\mathrm{q}_{\mathrm{eq}}\left(\mathrm{mg} \mathrm{g}^{-1}\right)$ & $61,66 \pm 1,45$ & $50,24 \pm 1,22$ & $53,72 \pm 1,38$ \\
\hline & & $\mathrm{K}_{2}\left(\mathrm{~g} \mathrm{mg}^{-1} \mathrm{~min}^{-1}\right)$ & $2,06.10^{-3} \pm 0,0003$ & $1,22.10^{-3} \pm 0,0001$ & $0,00313 \pm 0,0006$ \\
\hline & & $\mathrm{R}^{2}$ & 0,9551 & 0,9740 & 0,9684 \\
\hline \multirow{6}{*}{ CADS04 } & \multirow{3}{*}{$\begin{array}{c}\text { Pseudo-primeira } \\
\text { ordem }\end{array}$} & $\mathrm{q}_{\mathrm{eq}}\left(\mathrm{mg} \mathrm{g}^{-1}\right)$ & $59,06 \pm 1,85$ & $54,05 \pm 1,75$ & $54,63 \pm 1,67$ \\
\hline & & $\mathrm{K}_{1}\left(\min ^{-1}\right)$ & $0,0968 \pm 0,018$ & $0,0381 \pm 0,00508$ & $0,0818 \pm 0,018$ \\
\hline & & $\mathrm{R}^{2}$ & 0,8877 & 0,9254 & 0,8270 \\
\hline & \multirow{3}{*}{$\begin{array}{l}\text { Pseudo-segunda } \\
\text { ordem }\end{array}$} & $\mathrm{q}_{\mathrm{eq}}\left(\mathrm{mg} \mathrm{g}^{-1}\right)$ & $63,29 \pm 1,26$ & $58,37 \pm 1,33$ & $58,55 \pm 1,67$ \\
\hline & & $\mathrm{K}_{2}\left(\mathrm{~g} \mathrm{mg}^{-1} \mathrm{~min}^{-1}\right)$ & $0,0022 \pm 0,00033$ & $9,88 \cdot 10^{-4} \pm 1,3 \cdot 10^{-4}$ & $0,0021 \pm 0,00044$ \\
\hline & & $\mathrm{R}^{2}$ & 0,9668 & 0,9620 & 0,9357 \\
\hline
\end{tabular}

Fonte: autor (2015)

Na Tabela 3 observa-se a quantidade máxima adsorvida para ambos os carvões. Em ambos os pH estudados, a quantidade máxima adsorvida foi superior para o CADSO4, devido à presença de grupos funcionais ácidos presentes na superfície do carvão ativado.

Nota-se que em solução ácida, $\mathrm{pH}=2$, ocorreu a maior remoção de paracetamol, tanto para o CAD e para CADSO4. Nota-se que a maior remoção ocorreu no CADSO4 em que a quantidade máxima adsorvida foi de $65,16 \mathrm{mg} \cdot \mathrm{g}^{-1}$.

Tabela 3- Quantidade máxima adsorvida para CAD e CADSO4

\begin{tabular}{ccc}
\hline & $\mathrm{q}_{\text {máx }}$ & $\mathrm{q}_{\text {máx }}$ \\
Amostra & $\mathrm{CAD}\left(\mathrm{mg}^{-1}\right)$ & CADSO4(mg.g $\left.{ }^{-1}\right)$ \\
\hline $\mathrm{pH}=2$ & 62,36 & 65,16 \\
$\mathrm{pH}=\mathrm{pH} \mathrm{pcz}_{\mathrm{pz}}$ & 49,61 & 58,18 \\
$\mathrm{pH}=10$ & 55,70 & 61,40 \\
\hline
\end{tabular}

adsorção de fármacos como o Ibuprofeno (ANIA, et al 2010), corantes como o azul de metileno (ALMEIDA, et al. 2011), fenol (DIN, HAMEED e AHMAD, 2009) e metais, como o chumbo (NADEEM et al. 2006) e cromo (IV) ( PARK e JANG, 2002). Este tipo de modelo cinético indica interações envolvendo o processo de quimissorção (McKAY e HO, 1999).

\subsection{Isoterma de Adsorção de Paracetamol}

As isotermas de adsorção de paracetamol nos carvões ativados estudados em solução de $\mathrm{pH}=2, \mathrm{pH}=\mathrm{pHpcz}$ e $\mathrm{pH}=10$ e os ajustes da isoterma de Langmuir são apresentados nas Figuras 6 e 7. 
Figura 6- Isoterma de Adsorção e ajuste de Langmuir para CAD

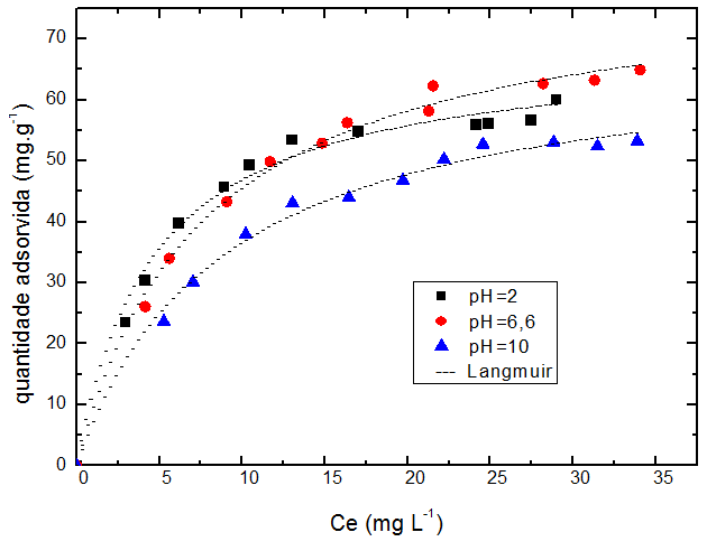

Fonte: Autor (2015)

Figura 7- Isoterma de Adsorção e ajuste de Langmuir para CADSO4

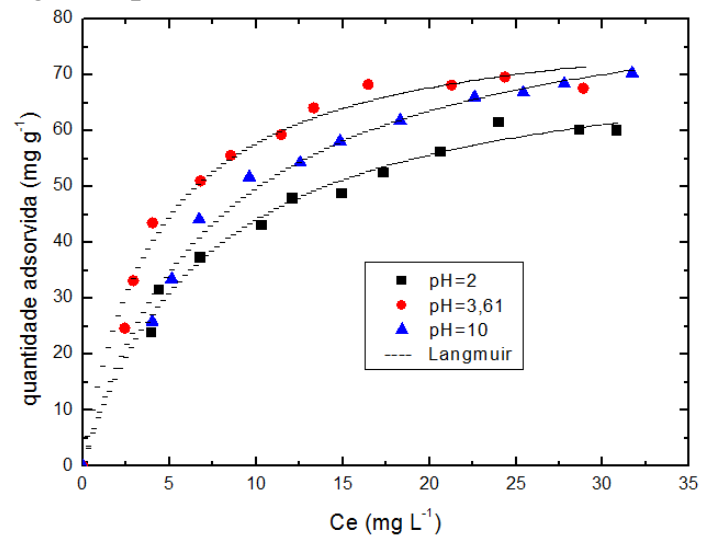

Fonte: Autor (2015)
As isotermas obtidas por ambos adsorventes são do tipo $\mathrm{L}$ de acordo com a classificação de GILES et al. (1960), com curvatura côncava em relação à abcissa. Neste tipo de isoterma ocorre a diminuição dos sítios de adsorção disponíveis quando a concentração da solução aumenta. A isoterma do tipo L tem como característica de sistema em que o adsorbato apresenta alta afinidade para $\mathrm{o}$ adsorvente.

Analisando as Figuras 6 e 7, nota-se que para a maior quantidade de paracetamol adsorvida ocorreu em $\mathrm{pH}_{\mathrm{pcz}}$ e $\mathrm{pH}=10$ para o $\mathrm{CAD}$ e CADSO4, respectivamente.

Tabela 5- Quantidade máxima adsorvida nas isotermas de CAD e CADSO4

\begin{tabular}{ccc}
\hline & $\mathrm{q}_{\text {máx }}$ & $\mathrm{q}_{\text {máx }}$ \\
Amostra & $\mathrm{CAD}\left(\mathrm{mg} \mathrm{g}^{-1}\right)$ & CADSO4 $\left(\mathrm{mg} \mathrm{g}^{-1}\right)$ \\
\hline $\mathrm{pH}=2$ & 60,20 & 61,66 \\
$\mathrm{pH}=\mathrm{pH} \mathrm{pcz}_{\mathrm{p}}$ & 64,95 & 69,62 \\
$\mathrm{pH}=10$ & 53,27 & 70,28 \\
\hline
\end{tabular}

Fonte: Autor (2015)

Os dados experimentais foram analisados nos modelos de Langmuir e Freundlich e os parâmetros obtidos estão dispostos na Tabela 6 .

Em todos os casos, os dados experimentais se ajustaram melhor ao modelo de Langmuir, uma vez que os valores de $\mathrm{R}^{2}$ foram mais próximos a 1 e os parâmetros apresentaram baixos valores de desvios.

Tabela 6- Parâmetros das Isotermas de Langmuir e de Freundlich para CAD e CADSO4

\begin{tabular}{cccccc}
\hline Carvão Ativado & Isoterma & Parâmetros & $\mathrm{pH}=2$ & $\mathrm{pH}=\mathrm{pH}_{\mathrm{pcz}}$ & $\mathrm{pH}=10$ \\
\hline & \multirow{2}{*}{ Langmuir } & $\mathrm{q}_{\max }\left(\mathrm{mg} \mathrm{g}^{-1}\right)$ & $68,86 \pm 1,75$ & $80,60 \pm 1,82$ & $68,97 \pm 1,99$ \\
& & $\mathrm{~b}\left(\mathrm{~L} \mathrm{mg}^{-1}\right)$ & $0,213 \pm 0,020$ & $0,129 \pm 0,009$ & $0,112 \pm 0,010$ \\
& & $\mathrm{R}^{2}$ & 0,9890 & 0,9944 & 0,9921 \\
& & $\mathrm{~K}_{\mathrm{F}}\left[\mathrm{mg} \mathrm{g}^{-1}\left(\mathrm{mg} \mathrm{L}^{-1}\right)^{-1 / n}\right]$ & $22,14 \pm 2,35$ & $19,37 \pm 2,002$ & $15,35 \pm 1,65$ \\
& \multirow{2}{*}{ Freundlich } & $1 / \mathrm{n}^{2}$ & 0,301 & 0,357 & 0,369 \\
& & $\mathrm{R}^{2}$ & 0,9528 & 0,9707 & 0,9736 \\
\hline \multirow{4}{*}{ Langmuir } & $\mathrm{q}_{\max }\left(\mathrm{mg} \mathrm{g}^{-1}\right)$ & $75,78 \pm 2,48$ & $81,78 \pm 2,43$ & $88,22 \pm 2,63$ \\
& & $\mathrm{~K}_{\mathrm{L}}\left(\mathrm{L} \mathrm{mg}^{-1}\right)$ & $0,138 \pm 0,013$ & $0,239 \pm 0,025$ & $0,128 \pm 0,011$ \\
& & $\mathrm{R}^{2}$ & 0,9884 & 0,9854 & 0,9909 \\
& \multirow{2}{*}{ CADSO4 } & $\mathrm{K}_{\mathrm{F}}\left[\mathrm{mg} \mathrm{g}^{-1}\left(\mathrm{mg} \mathrm{L}^{-1}\right)^{-1 / n}\right]$ & $17,92 \pm 1,54$ & $26,49 \pm 2,85$ & $19,83 \pm 2,086$ \\
& \multirow{2}{*}{ Freundlich } & $1 / \mathrm{n}^{2}$ & 0,371 & 0,310 & 0,380 \\
& & $\mathrm{R}^{2}$ & 0,9773 & 0,9422 & 0,9683 \\
\hline
\end{tabular}

Fonte: Autor (2015) 
Ao comparar a quantidade máxima adsorvida, Tabela 5, com a quantidade máxima de Langmuir, Tabela 6 , nota-se que para ambos os casos a quantidade máxima adsorvida não foi experimentalmente atingida.

Os valores de $K_{L}$, apresentados na Tabela 6, mostram que o carvão CADSO4 apresentou maior afinidade para a adsorção de paracetamol do que o CAD já que as isotermas de CADSO4 apresentam maiores valores de $\mathrm{K}_{\mathrm{L}}$ para os três valores de $\mathrm{pH}$ estudados. Portanto, cada molécula de paracetamol está ligada a somente um sítio especifíco dos carvões, e não ocorrendo interações entre as moléculas de paracetamol, assim formando uma monocamada, caracteristica de isotermas que seguem $o$ modelo de Langmuir.

Após a funcionalização as moléculas de paracetamol pode-se interagir com grupos ácidos como os carboxílicos, fenólicos e lactonicos presentes na superfície do carvão ativado funcionalizado, assim a quantidade máxima adsorvida é maior, como representado pelas tabelas 3 e 5

\subsection{CONCLUSÃO}

Os resultados apresentados nesse estudo mostram que após a funcionalização com ácido sulfúrico as características texturais e químicas são modificadas. Ocorre o decréscimo da área de superfície especifica, do volume de poros e de microporos e o aumento dos grupos ácidos presentes na superfície do carvão. A capacidade de adsorção de paracetamol foi superior para $\mathrm{o}$ carvão ativado funcionalizado. Tanto para CAD e CADSO4, o modelo de pseudosegunda ordem e a equação de Langmuir podem representar os dados experimentais cinéticos e de equilíbrio, respectivamente, assim caracterizando uma adsorção em multicamada.

\section{NOMENCLATURA}

CAD- Carvão ativado de Dendê

CADSO4 - Carvão ativado de Dendê funcionalizado com ácido sulfúrico qt- Quantidade adsorvida no equilíbrio t (mg $\left.\mathrm{g}^{-1}\right)$

$\mathrm{c}_{0}$ - $\mathrm{c}_{\mathrm{i}}$ - Concentração inicial e final $\left(\mathrm{mg} \mathrm{L}^{-1}\right)$ $\mathrm{pH}_{\mathrm{pcz}}-\mathrm{pH}$ de ponto de carga zero

$\mathrm{K}_{1}$ - Constante cinética de pseudo-primeira ordem $\left(\mathrm{min}^{-1}\right)$

qeq- $_{\text {- }}$ Quantidade adsorvida no equilíbrio (mg $\left.\mathrm{g}^{-1}\right)$

$\mathrm{K}_{2}$ - Constante cinética de pseudo-segunda ordem (min $\mathrm{g} \mathrm{mg}^{-1}$ )

${\text { q } \max ^{-}}$quantidade máxima adsorvida pelo modelo de Langmuir ( $\mathrm{mg} \mathrm{g}^{-1}$ )

$\mathrm{C}_{\mathrm{eq}}$ - Concentração de equilíbrio $\left(\mathrm{mg} \mathrm{g}^{-1}\right)$

$\mathrm{K}_{\mathrm{L}^{-}}$Constante de Langmuir $\left(\mathrm{L} \mathrm{mg}^{-1}\right)$

$\mathrm{K}_{\mathrm{F}^{-}}$Constante de capacidade de adsorção de Freundlich $\left[\mathrm{mg} \mathrm{g}^{-1}\left(\mathrm{mg} \mathrm{L}^{-1}\right)^{-1 / n}\right.$ ]

n- Intensidade de adsorção

\section{REFERÊNCIAS}

ALMEIRA, Vitor C.; MORAES, Juliana C.G.; SILVA, Tais L.; MARTINS, Alessandro C.; GUILHERME, Marcos R.; KUNITA, Marcos H.; NOGAMI, Eurica M.; VARGAS, Alexandro M.M.; CAZETTA, André L.; $\mathrm{NaOH}$-activated carbon of high surface area produced from coconut shell: Kinetics and equilibrium studies from the methylene blue adsorption. Chemical Engineering Journal. v.174 p.117-125, 2011

BACCAR, R.; SARRÀ, M.; BOUZID, J.; FEKI, M.; BLÁNQUEZ, P.; Removal of pharmaceutical compounds by activated carbon prepared from agricultural by-product. Chemical Engineering Journal. v.211-212, p.310-317, 2012

BARCELÓ ,D.; GENTILI, A.; PASTORI,F.; QUINTANA, J.B.; RODIL, R.; GANS, O.; 
KORMALI, P.; ZUCCATO, E.; TERNES, T.A.; FINKI, G.; KNEPPER, T.; MULLER, J., ALENCASTRO, F.DE; BUDZINSKI, H.; MENACH, K.L.; LOOS, R.; OSVALD, P.; HEATH, E.; MARTINEZ, E.; KOSJEK, T.; GROS, M.; PETROVIC, M.; FARRÉ M.; First interlaboratory exercise on non-steroidal anti-inflmmatory drugs analysis in enviranmental samples. Talanta. v.76, p.580590, 2008

BOEHM, H.P. Some aspects of the surface chemistry of carbon blacks and other carbons. Carbon. v. 5, p. 759-769, 1994

COUTO-JR, Osorio M.; MATOS, Inês; FONSECA, Isabel M.; ARROYO, Pedro A.; SILVA, Edson A.; BARROS, Maria A. S. D. Effect of Solution $\mathrm{pH}$ and Influence of Water Hardness on Caffeine Adsorption onto Activated Carbons. Can. J. Chem. Eng. v.93, p.68-77, 2015

DIN, Azam T.M.; HAMEED, B.H.; AHMAD, Abdul L.; Batch adsorption of phenol onto physiochemical-activated coconut shell. Journal of Hazardous Materials. V.161, p.1522-1529, 2009

DUCLAUX, Laurent; GUEDIDI, Hanen; REINERT Laurence; LÈVÊQUE, Jean-Marc; SONEDA, Yasushi, BELLAKHAL, Nizar. THe effects of the surface oxidation of activated carbon, solution $\mathrm{pH}$ and the temperature on adsorption of ibuprofen. Carbon, v.54, p.432-443,2013

FENT, Karl, WESTON, Anna A., CAMINADA, Daniel. Ecotoxicolody of human pharmaceuticals. Aquatic Toxicology. v.76, p.122-159,2006

FREUNDLICH, H. Adsoption in Solution. Phys. Chemie. V. 57, p.384-410. 1906
GILES, C.H.; MACEWAN, T.H.; NAKHWA, S.N.; SMITH, D.Studies in Adsorption. Part XI.* A System of Classi\$cation of Solution Adsorption Isotherms, and its Use in Diagnosis of Adsorption Mechanisms and in Measurement of Specific Surface Areas of Solids.39733993, 1960

HOTZA, Dachamir; IGLESIAS, Alberto; LLÓPIZ, Julio C.; TOBELLA, Jorge; GARCIA, Raquel; TACORONTE, Juan; REY-MAFULL, Carlos A. Comparative study of the adsorption of acetaminophen on activated carbons in simulated gastric fluid. SpringerPlus, v.3, p.48-60, 2014

HUANG, Chen-Chia; LI, Hong-Song; CHEN, Chien-Hung. Effect of surface acidic oxides of activated carbon on adsorption of ammonia. Journal of Hazardous Materials. v.159, p.523-527, 2008

LAGERGREN, S. Zur theorie der sogenannten adsorption gel`ster stoffe, Kungliga Svenska Vetenskapsakademiens. Handlingar. v.24 p.1-39 1898

LANGMUIR, Irving. The Adsorption of Gases on Plane Surfaces of Glass, Mica and Platinum. J. Am. Chem Soc. V.40, p.13611403, 1918

LI, Liqing; LIU, Zheng; TANG, Lin; SHI, Rui; GU, Qingwei; LIANG, Xing, YAO, Xiaolong. Investigation of adsorption performance on 1,2-dichloroethane by heat and acid modified activated carbon. Journal of Environmental Chemical Engineering. v.1, p. 131-136, 2013

LIU, Junxin; LIU, Suqin; LI, Lin. Surface modification of coconut shell based activated carbon for the improvement of hydrophobic VOC removal. Journal of Hazardous Materials. v.192, p. 683-690, 2011 
MASTRAGOSTINO, Marina; SEMERARO, Domenica; BENINATI, Sabina. Adsorption of Paracetamol and Acetylsalicylic Acid onto commercial activated carbons. Adsorption Science \& Technology. v.26, p.721-734, 2009

MCKAY, G.;HO, Y.S.; Pseudo-Second Order Model for Sorption Processes. Process Biochemistry. v.34 p. 451-465. 1999

MESTRE, Ana S.; PIRES, Ricardo A.; AROSO, Ivo; FERNANDES, Emanuel M.; PINTO, Moisés L.; REIS, Rui L.; ANDRADE, Marta A.; PIRES, João; SILVA, Susana P.; CARVALHO, Ana P. Activated carbons prepared from industrial pre-treated cork: Sustainable adsorbents for pharmaceutical compounds removal. Chemical Engineering Journal. v.253, p.408-417, 2014

MOREIRA, Regina F. P. M.; JOSÉ, Humberto J.; YAMANAKA, Leonardo Y.; TAMBOSI, José L.; SCHRODER, Horst F. Recent Research data on the removal of pharmaceutical from sewage treatment plants (STP). Química Nova, v.33, p.411-420,2010

MUIR N.; NICHOLS, J. D.; STILLINGS, M. R.; SYKES, J. Comparative bioavailability of aspirin and paracetamol. Curr. Med. Res., v.13, 491-500, 1997

NADEEM, Muhammad; MAHMOOD, A.; SHAHID, S.A.; SHAH, S.S; KHALID, A.M.; McKAY, G. Sorption of lead from aqueous solution by chemically modified carbon adsorbents. Journal of Hazardous Materials. v. 138, p. 604-613, 2006.

PARK, Soo-Jin; JANG, Yu-Sin. Pore Structure and Surface Properties of Chemically Modified Activated Carbons for Adsorption Mechanism and Rate of
Cr(VI).Journal of Colloid and Interface Science. v.249, p. 458-463, 2002

REGALBUTO J.R.; PARK J. A simple, accurate determination of oxide PZC and the strong buffering effect of oxide surfaces at incipient wetness. J. Colloid Interface Sci. v.175,p.239-252 1995

SARKAR, Ujjaini; BANERJEE, Debasree; CHAKRABORTY, Sayantani; ROY, Desasri. Removal of a cationic bisbiguanide using Functionalized Activated Carbons (FACs). Process Safety and Environmental Protection. v.92, p.957-972, 2014

TERZYK, Artur p.; RYCHLICKI, Gerhard. The influence of activated carbon surface chemical composition on the adsorption of acetaninophen (paracetamol) in vitro. The temperature dependece of adsorption at the neutral pH. Colloids and Surfaces. v.163, p. 135-150, 2000

VILLAESCUSA, Isabel; FIOL, Núria; POCH, Jordi; BIANCHI Antonio; BAZZICALUPI, Carla. Mechanism of paracetamol removal by vegetable wastes: The contribution of $\pi-\pi$ interactions, hydrogen bonding and hydrophobic effect.

Desalination, v.270, p.135-142,2011

\section{AGRADECIMENTOS}

Os autores agradecem ao Departamento de Engenharia Química e a Capes pelo suporte financeiro. 